\section{A APLICAÇÃO DE TÉCNICAS DE IMPOSIÇÃO DE MÃOS NO CÂNCER: REVISÃO SISTEMÁTICA DA LITERATURA}

\section{THE APPLICATION OF TECHINIQUE OF IMPOSITION OF HANDS IN THE CÂNCER: SYSTEMATIC LITERATURE REVIEW}

\section{RESUMO}

O objetivo é analisar, por revisão sistemática da literatura, o uso e o efeito de técnicas de imposição de mãos (TIM): Reiki, Therapeutic Touch e Healing Touch, no câncer. Foram realizadas buscas no PubMed/MedLine, Lilacs, Web of Science, Ebsco e Embase, em outubro de 2013, selecionados 19 artigos e analisados em relação à: metodologia, objeto de estudo, fatores de impacto dos jornais, países de desenvolvimento, ano de publicação e resultados alcançados. A literatura mostra: predominância de metodologia quantitativa; uso das TIM no cuidado de sintomas de ansiedade ou dor provocados pelo câncer; predomínio de publicações no EUA; publicação do primeiro artigo indexado em 1999; publicações em jornais de baixo ou nenhum fator de impacto conforme o Journal Citation Reports; e predominância de estudos com resultados estatisticamente significativos. Apesar de a maioria dos estudos apresentarem resultados positivos, nenhum avaliou o impacto das TIM no foco da doença, o que gera uma lacuna neste campo do conhecimento e a secundarização destas técnicas como recurso de tratamento.

\section{PALAVRAS-CHAVE}

Reiki.

Therapeutic Touch.

Câncer.

Neoplasia.
Pedro Mourão Roxo da Motta

- Fisioterapeuta, Mestre - Faculdade de Ciências Médicas da UNICAMP - Campinas, SP, Brasil

Nelson Filice de Barros

- Sociólogo, Pós-Doutor, Coordenador do LAPACIS - Laboratório de Práticas Alternativas Complementarese

Integrativas em Saúde - Faculdade de Ciências Médicas da UNICAMP - Campinas, SP, Brasil

CORRESP ON DEN T E

LAPACIS - Laboratório de Práticas Alternativas Complementares e Integrativas em Saúde.

Faculdade de Ciências Médicas da UNICAMP - Departamento de Saúde Coletiva.

Rua: Tessália Vieira de Camargo, 126 Cidade Universitária "Zeferino Vaz" Campinas - SP - Brasil - CEP: 13083-887 Fone (19) 3521.9240 - 3521.8036

E - M A I L

terapeutapedromotta@hotmail.com

Recebido: 24/04/2014

Aprovado: 11/07/2014 


\begin{abstract}
ABSCTRACT
The objective is to analyze, according to a systematic literature review, the use and the effect of techniques of laying on hands (TLH): Reiki, Therapeutic Touch and Healing Touch in cancer. Searches were performed in PubMed/Medline, Lilacs, Web of Science, Embase and Ebsco, in October 2013, 19 articles selected and analyzed following issues: Methodology, object of study, impact factors of journals, developing countries, year of publication and results. The literature shows: predominance of quantitative methodology, use of TLH in the care of symptoms of anxiety or pain caused by cancer; predominance of publications in the U.S; publication of the first indexed article in 1999; publications with low or no impact factor as Journal Citation Reports; and predominance of studies with statistically significant results . Although most studies report positive results, none have evaluated the impact of TLH on the focus of disease, which creates a gap in this field of knowledge and sidelines these techniques as a treatment resource.
\end{abstract}

KEYWORDS: Reiki, therapeutic touch, cancer, neoplasms.

\section{INTRODUÇÃO}

Reiki, Therapeutic Touch (TT) e Healing Touch (HT) são Técnicas de Imposição de Mãos (TIM) que trabalham e visam o reequilíbrio e harmonização do sistema energético do ser humano. $O$ Reiki é uma técnica de canalização de energia vital através de símbolos e sons sagrados, que atuam como chave para canalização da energia pelo terapeuta para o paciente. Apesar desta energia ainda não ser qualificada pela física moderna, alguns autores relatam a existência de um biocampo, que seria o campo de energia (CE) intrínseco a todos os seres vivos, chamado também de campo quântico, clássico e não clássico ${ }^{1}$.

A palavra "Rei" em japonês significa universal, e "Ki" é o termo usado para descrever a energia vital que carrega a força da vida. Da união da palavra "Rei-universal" com a palavra "Ki-energia vital" temos "energia vital universal". É comumente aplicado por meio do toque das mãos no corpo do paciente em posições específicas, ou à distância, mesmo a pessoa se encontrando em outro lugar do planeta ${ }^{2}$.

Durante uma aplicação de Reiki, não é necessário o direcionamento da energia ou a intenção de curar por parte do terapeuta, pois, pelo atributo "Rei" da Energia, o "Ki” se direciona para onde é necessário, isto atenua a preocupação do terapeuta em relação à perda de sua energia, pois ao passar pelo seu corpo, antes de chegar ao paciente, beneficia também o terapeuta.
Mikao Usui é citado na literatura pesquisada como o redescobridor do Reiki, porém há uma diversidade de histórias diferentes contadas devido às inúmeras adaptações feitas para introduzir essa prática na cultura ocidental ${ }^{3}$. Alguns autores relatam que o Reiki tem sua origem há 3000 anos A.C. nas escrituras sagradas do Tibet (Sutras Tibetanos). Porém, até o presente momento, não existem provas empíricas que sustentem essa afirmação.

Após redescobrir o Reiki, Mikao Usui, inicia Chujiro Hayashi no ano de 1925, como Mestre Professor. Hayashi, que era médico aposentado da marinha, leva essa terapia para sua clínica em Tóquio. Em 1939 Hayashi inicia Hawayo Takata como Mestre Professora, que, por sua vez, trabalha intensamente o Reiki no Hawai, Japão e Estados Unidos, formando 22 Mestres entre homens e mulheres. $\mathrm{O}$ Ocidente veio conhecer o Reiki em meados de 1970 e o Brasil em 1982.

O Reiki, com sua chegada no Ocidente, sofreu inúmeras alterações para se adaptar a esta cultura. Quando ele foi redescoberto por Mikao Usui era aplicado de maneira mais intuitiva; não existia um protocolo de tratamento como atualmente no Ocidente. Ao invés disso, existia um método de diagnóstico específco, em que os alunos treinavam intensamente captar distúrbios energéticos no corpo para reequilibrá-los. Sem contar que a formação de um Reikiano, por ve- 
zes, durava a vida inteira, já que um aluno poderia levar em média 10 anos para passar de um nível para o outro, após longo período de prática e dedicação. Porém, atualmente, a mudança de nível ocorre quando a pessoa tem disponibilidade de tempo e dinheiro para custear o curso do próximo nível 5 .

O Reiki tem um papel importante no desenvolvimento da consciência do autocuidado, pois antes de poder aplicar Reiki em outras pessoas é necessário que o iniciado se autoaplique diariamente uma hora e dez minutos, durante 21 dias, como processo de purificação energética. Isto leva ao desenvolvimento do autocuidado, por parte do terapeuta de Reiki, o que pode criar, de maneira sutil, esta consciência coletiva na cultura.

O National Center of Complementary and Alternative Medicine (NCCAM) dos Estados Unidos da América caracteriza o Reiki como uma terapia complementar ${ }^{6}$. No Brasil, dentre as Práticas Integrativas e Complementares (PIC) encontradas pelo Ministério da Saúde em 2004, o Reiki foi identificado como a mais utilizada no Sistema Único de Saúde (SUS) $(25,6 \%)^{7}$. Isso se deve a inúmeros motivos, dentre eles o fato de não ter registro de contra-indicação, não necessitar de procedimentos invasivos, não necessitar de esforço físico e possibilitar uma prática de restabelecimento de energia de fácil e simples acesso.

O TT foi criado pela enfermeira Dolores Krieger em 1972 e está baseado no reequilíbrio e harmonização do CE humano. Nesta prática o ser humano é considerado um sistema dinâmico de energia que interliga os aspectos físicos, emocionais, mentais e espirituais. Trabalhando no CE o terapeuta interfere nestas diferentes dimensões facilitando, assim, a cura ${ }^{8}$.

Os passos para a realização do TT são: a) centralização, momento em que o terapeuta se centra na sensibilidade de suas mãos a fim de perceber o outro e curá-lo; b) acesso e avaliação do CE do paciente, o terapeuta toca o $\mathrm{CE}$ do paciente colocando suas mãos numa distância de $6 \mathrm{a} 12 \mathrm{~cm}$ da pele e percorre-o percebendo qualquer desarmonia existente; c) tratamento e modulação do $C E$, o terapeuta deve restituir o padrão natural do $\mathrm{CE}$; d) balanceamento final e estabelecimento do fluxo energético, o terapeu- ta mantém o CE do paciente homogêneo e harmônico; e) avaliação, o terapeuta reavalia os achados no CE do paciente para comparar os achados com os da próxima sessão. Além destes procedimentos é necessário que o terapeuta esteja saudável, centralizado e equilibrado do ponto de vista energético e com a intenção de ajudar o paciente $e^{9,10,11}$.

HT é um tratamento energético criado com o fim de expandir a conexão inter-relacional entre enfermeiras e pacientes, em 1989, por Janet Mentgen, uma enfermeira que trabalhou 43 anos observando essa conexão. Para se aplicar o HT é necessário que o terapeuta esteja centrado e em paz, então ele utilizará suas mãos sobre e no corpo do paciente, para acessar e determinar áreas de desequilíbrio de energia, percebidas como mudanças na temperatura, textura, ou vibração. Com a aplicação do HT pretende-se restaurar a harmonia e o equilíbrio energético facilitando assim a "autopoiese"12.

Há inúmeras distinções entre essas técnicas, pois o Reiki, ao que tudo indica, é uma técnica milenar que tem sua origem no Oriente, utiliza de símbolos e sons sagrados para a canalização de energia vital, e tem como premissa um processo de 21 dias de autoaplicação, para o iniciado poder aplicar em outras pessoas. O TT e o HT têm como premissa que o terapeuta esteja centrado e em paz, com a intenção de curar o paciente para ser aplicado. Além disso, diferentemente do Reiki, foram criados no Ocidente recentemente, trabalham com o CE do ser humano, não utilizam de símbolos nem sons, apenas a percepção energética das mãos do terapeuta para harmonizar o $\mathrm{CE}$ do paciente. Apesar das distinções entre as técnicas, elas se assemelham pois todas trabalham de alguma forma com o autocuidado do terapeuta.

As TIM são uma versão contemporânea de práticas ancestrais de cura pelas mãos, têm sido utilizadas em diversas circunstâncias e com diversos propósitos com o intuito de promover relaxamento e melhora da qualidade de vida, ou então para tratar os sintomas secundários ocasionados por doenças e seus respectivos tratamentos ${ }^{13}$.

A pesquisa sobre o uso das TIM no tratamento de doenças como, por exemplo, o câncer tem cresci- 
do nos últimos anos. As produções científicas sobre estas técnicas no câncer procuram responder à necessidade de disponibilizar informações nos meios de comunicação, principalmente em relação ao custo dos tratamentos e a toxicologia das intervenções em dois momentos: quando são utilizadas com tratamentos convencionais ou utilizadas sozinhas ${ }^{14}$.

O câncer é uma doença crônica que envolve uma série de mudanças e dificuldades, afetando os pacientes em seus aspectos biológico, psíquico e social. O centro Nacional do Câncer dos Estados Unidos encontrou que entre $9 \%$ e $91 \%$ dos pacientes oncológicos usam alguma forma de PIC ${ }^{15}$. No Brasil o câncer é a segunda maior causa de morte, as longas filas para o tratamento e a demora pela assistência são os maiores fatores pelos índices de mortalidade. Existem poucas pesquisas sobre o uso das PIC no tratamento oncológico no Brasil, um estudo sustenta que $63 \%$ dos pacientes com câncer utilizam alguma forma de PIC ${ }^{16}$.

Crescentes evidências suportam a veracidade da conexão entre mente e corpo, e sugerem o importante benefício de intervenções nesta dimensão do cuidado no auxílio do tratamento oncológico ${ }^{15}$. As TIM, por sua vez, têm sido aplicadas aos pacientes com câncer como terapia complementar em adição à terapia convencional, usualmente para tratar os sintomas secundários e colaterais ocasionados pela quimioterapia e radioterapia, como: dor, stress e ansiedade ${ }^{12}$.

Este artigo tem como objetivo analisar sistematicamente a literatura sobre o uso e efeito das TIM no câncer, por meio da análise do objeto de estudo, da metodologia utilizada na investigação, do fator de impacto do jornal em que o artigo foi publicado, do país em que o estudo foi desenvolvido, do ano de publicação e dos resultados do uso das TIM no cuidado do câncer.

\section{METODOLOGIA}

Pela necessidade de compilação das evidências científicas disponíveis, construindo assim conclusões advindas de diferentes fontes, o método selecionado neste trabalho foi o da Revisão Sistemática da Literatura (RSL). Após a escolha do tema de interesse foi realizada a busca e seleção dos artigos conforme consta na Figura 1. Posteriormente. os trabalhos selecionados foram analisados criticamente conforme os objetivos do estudo ${ }^{17}$. Foram adotados como critérios de inclusão:

»artigos de qualquer data e nacionalidade.

» artigos de língua inglesa ou portuguesa que continham em seu título ou resumo as palavras chave: "Reiki and Cancer"; "Therapeutic Touch and Cancer"; "Healing Touch and Cancer".

Foram adotados como critérios de exclusão:

» artigos que relatem experimentos que não foram realizados em seres humanos.

» artigos que não eram sobre o câncer.

» artigos indisponíveis na íntegra nas diferentes bases de dados.

» artigos de revisão.

» artigos que não relatavam o uso/efeito das TIM no câncer.

» artigos que fossem duplicatas.

»artigos de revisão de literatura.

Figura 2 - Critérios de exclusão e seleção dos artigos

\section{BUSCA E SELEÇÃO DOS ARTIGOS}

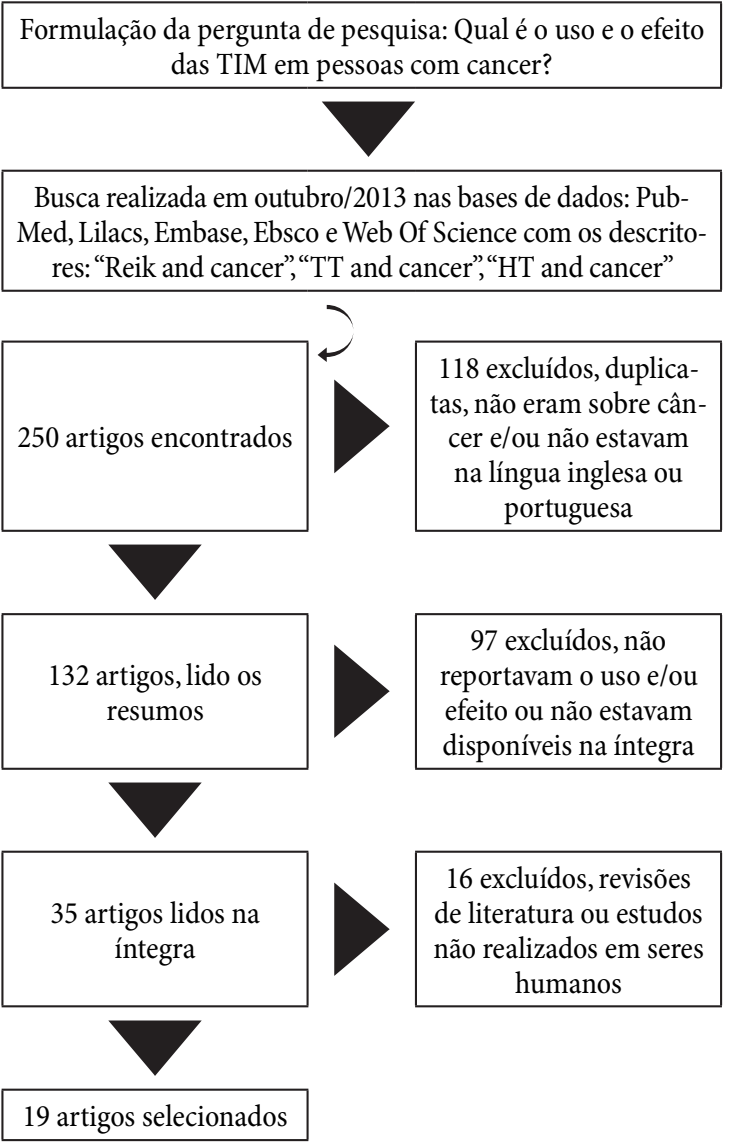

Formulação da pergunta de pesquisa: Qual é o uso e o efeito das TIM em pessoas com cancer?

Busca realizada em outubro/2013 nas bases de dados: Pubed, Lilacs, Embase, Ebsco e Web Of Science com os descrito- 


\section{RESULTADOS}

A primeira publicação indexada sobre a aplicação das TIM no câncer data de 1999, o que revela tratar-se de um campo relativamente novo de pesquisa. Além disso, conforme o quadro 1, 2, e 3 existe uma concentração de estudos das TIM, 13 dos 19 artigos, publicados a partir de 2007, o que pode ser um indício de que a comunidade científica está se interessando mais por estas terapias no cuidado de pacientes com câncer.

Dos 19 artigos analisados, 13 foram publicados nos EUA, dos quais sete

obtiveram resultados estatisticamente não significativos e 6 significativos. No Canadá, foram publicados 3 artigos, Inglaterra, Irã e Brasil publicaram um cada um, todos com resultados significativos. Os dados demonstram a predominância de estudos na América do Norte, 16, em sua maior parte nos EUA, 13 , o que pode ser explicado pelo alto investimento que este país faz em pesquisas em PIC, sobretudo após a criação do NCCAM em 1998.

Os estudos apresentam uma predominância metodológica quantitativa, 15 entre 19, destes 9 são experimentais, 2 quase experimentais, 4 observacionais e 4 qualitativos. A ênfase na metodologia quantitativa pode constituir um viés de pesquisa em TIM, pois a aplicação destas técnicas é única e individual e a padronização intrínseca dos desenhos de pesquisa controlados e randomizados nem sempre se ajustam a esta lógica ${ }^{18}$.

Em relação ao FI dos jornais em que os artigos foram encontrados, observa-se que apenas 10 dos 19 foram publicados em jornais com descrição no Journal Citation Reports ${ }^{18}$. Destes, os três estudos com maior fator de impacto são os de; Kelly et a ${ }^{20} \mathrm{e}$ Catlin et a ${ }^{21}$ publicados no periódico Oncology Nursing Forum, e Olson et a ${ }^{22}$ publicado no Journal of Pain and Symptom Management, ambos com FI de 2,50. Após estes, os trabalhos com maior FI são os de; Fleisher et $\mathrm{al}^{23}$, FitzHenry et $\mathrm{al}^{24}$, Wong $\mathrm{J}$ et $\mathrm{al}^{25}$, Tsang et $\mathrm{al}^{26}$ e Post-White $\mathrm{J}$ et a $\mathrm{l}^{12}$, todos publicados no Integrative Cancer Therapies com FI de 2,35. Em seguida, se apresenta o artigo de Frank LS et a ${ }^{27}$ pu- blicado no periódico Pain medicine cujo FI é de 2,34. E o trabalho com o menor FI é o de Marcus et $\mathrm{al}^{28}$ publicado no American Journal Hospice Palliative Care cujo FI é de 1,23.

Apenas alguns artigos foram publicados em periódicos com descrição do FI e todos são considerados de baixo impacto. A possível interpretação proveniente deste dado é que há uma dificuldade dos pesquisadores em publicar pesquisas em TIM especificamente e PIC em geral em revista de maior visibilidade, o que reflete a marginalização deste campo de pesquisa em relação à hegemonia biomédica.

Dos 19 artigos selecionados, 12 obtiveram resultados significativos, destes 3 avaliaram os resultados da aplicação das TIM na fadiga, dor e ansiedade em pacientes com câncer; $2 \mathrm{em}$ pacientes com efeitos colaterais ocasionados pela quimioterapia; e 1: na qualidade de vida; na dor e uso de medicamentos; na dor e fadiga; em pacientes problemas de concentração mental em diferentes estágios da doença; em pacientes com ansiedade; em crianças com sintomas de dor, stress e fadiga; e em pacientes com sintomas de fadiga e náusea.

Sete estudos obtiveram resultados não significativos, 2 em relação ao uso das TIM aplicadas aos efeitos colaterais de pacientes recebendo quimioterapia, 2 em pacientes com câncer de mama e sintomas de ansiedade, depressão e fadiga, e 1: no bem-estar em pacientes com câncer de próstata; na ansiedade de mulheres passando por biópsia; na qualidade de vida e progressão da doença em mulheres com câncer de ovário. Apesar de 7 dos 19 estudos terem tido resultados não significativos, não houve estudos que apontam ação prejudicial das TIM, levantando a possibilidade de que estas práticas são seguras e sem contraindicação.

Nenhum estudo avaliou o impacto das TIM no foco da doença e sim nos sintomas secundários da mesma. Além disso, em geral, os artigos estudaram pacientes com câncer de qualquer tipo, ou não descreveram o tipo de câncer que os pacientes submetidos aos estudos tinham. Dos 19 artigos, apenas 6 descrevem o tipo de câncer que o paciente portava, 3 de mama, 1 de próstata, 1 de ovário e 1 com leuce- 
mia. Estes dados revelam a complexidade do estudo das TIM no câncer, já que, cada tipo tem suas atribuições e características singulares.

Nos resultados demonstrados no quadro 1, dos 19 artigos, 7 utilizaram o Reiki, sendo que 4 deles obtiveram resultados estatisticamente significativos e 3 não significativos. 0 quadro 2, constituído de 6 artigos, demonstra o uso e o efeito do TT aplicado ao cuidado de pacientes oncológicos, em que $5 \mathrm{ob}$ tiveram resultados estatisticamente significativos e 1 resultado não significativo. No quadro 3, estão apresentados os 6 estudos sobre o HT, sendo 3 com resultados estatisticamente significativos e 3 não significativos. É importante salientar que na maioria dos estudos o número de participantes foi baixo, o que pode ter influenciado os estudos com resultados estatisticamente não significativos.

Os instrumentos utilizados para verificar o efeito das TIM em pacientes com câncer foram variados: quatro autores utilizaram o Functional Assessment

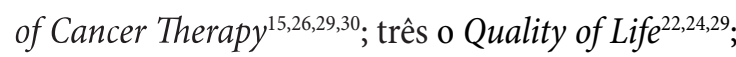
três o Epidemiological Studies Depression Scale ${ }^{15,30,31}$; três autores utilizaram a Escala Visual Analógica $^{22,27,32}$; dois Escala Auto Referida ${ }^{28,33}$; dois o Distress Thermometer ${ }^{23,34}$; dois autores utilizaram o State-Trait Anxiety Inventory ${ }^{31,35}$; e os instrumentos que seguem foram utilizados por apenas um autor cada: Well-Being Analog Scale ${ }^{21}$, Healing Touch Comfort Questionnaire $^{21}$, Rothen Fatigue Scale ${ }^{32}$, Telephone Interview Guide ${ }^{20}$, Feeling Thermometer ${ }^{25}$, My Fatigue Meter ${ }^{25}$, Spielberg State Anxiety Inventoryn ${ }^{15}$, Hospital Anxiety and Depression Scale ${ }^{31}$. Houve ainda autores que mensuraram os níveis do $\operatorname{IgA}$ sali$v^{2}{ }^{29}$, outros os sinais vitais, como pressão sanguínea, batimento cardíaco e respiratório ${ }^{12}$, além de alguns que construíram narrativas de pacientes ${ }^{36}$.

Devido ao pequeno número de artigos não é possível fazer distinções em relação às técnicas e seus resultados, os quais, apesar de em sua maioria serem positivos, são insuficientes para afirmações mais assertivas a respeito destas técnicas no cuidado de pessoas com câncer. Contudo, promovem um vislumbre dos possíveis efeitos benéficos das TIM.

Quadro 1: estudos do Reiki no câncer

\begin{tabular}{|c|c|c|c|}
\hline Referência & Técnica NS/DI/GE & Uso & Efeito \\
\hline $\begin{array}{l}\text { Fleisher et al } \\
2013\end{array}$ & $\begin{array}{l}\text { Reiki NS- N/I DI-20 GE- } \\
213\end{array}$ & $\begin{array}{l}\text { Ansiedade, stress, dor e fadiga } \\
\text { em pacientes com câncer }\end{array}$ & $\begin{array}{l}\text { Melhora significativa de todas as } \\
\text { variáveis }\end{array}$ \\
\hline $\begin{array}{l}\text { Marcus et al } \\
2013\end{array}$ & $\begin{array}{l}\text { Reiki NS- N/I DI- } 282 \\
\text { GE-145 }\end{array}$ & $\begin{array}{l}\text { Dor, stress e ansiedade em } \\
\text { pacientes com câncer }\end{array}$ & $\begin{array}{l}\text { Melhora significativa de todas as } \\
\text { variáveis }\end{array}$ \\
\hline $\begin{array}{l}\text { Catlin A; Taylor- } \\
\text { Ford RL; } 2011\end{array}$ & $\begin{array}{l}\text { Reiki NS-01/ DI- } 20 \text { GE- } \\
\text { 63/ GC-63/GP-63 }\end{array}$ & $\begin{array}{l}\text { Bem-estar em pacientes rece- } \\
\text { bendo quimioterapia }\end{array}$ & Não houve diferença significativa \\
\hline $\begin{array}{l}\text { Beard C; et all; } \\
2011\end{array}$ & $\begin{array}{l}\text { Reiki NS-16/ DI-50 GE- } \\
36 \text { GC-18 }\end{array}$ & $\begin{array}{l}\text { Bem-estar em pacientes com } \\
\text { câncer de próstata }\end{array}$ & Não houve diferença significativa \\
\hline $\begin{array}{l}\text { Tsang KL; etall } \\
2007\end{array}$ & $\begin{array}{l}\text { Reiki NS-07/DI-45 GE- } \\
\text { 08/GC-08 }\end{array}$ & $\begin{array}{l}\text { Fadiga, dor e ansiedade em } \\
\text { pessoas com câncer }\end{array}$ & $\begin{array}{l}\text { Melhora significativa de todas as } \\
\text { variáveis }\end{array}$ \\
\hline Potter PJ; 2007 & $\begin{array}{l}\text { Reiki NS-02/DI-54/GE- } \\
\text { 18/GC-17 }\end{array}$ & $\begin{array}{l}\text { Ansiedade em mulheres com } \\
\text { câncer de mama }\end{array}$ & Não houve diferença significativa \\
\hline $\begin{array}{l}\text { Olson K; et all } \\
2003\end{array}$ & $\begin{array}{l}\text { Reiki NS-02/DI-60 GE- } \\
\text { 11/GC-13 }\end{array}$ & $\begin{array}{l}\text { Dor e qualidade de vida em } \\
\text { pacientes oncológicos }\end{array}$ & $\begin{array}{l}\text { Melhora significativa de todas as } \\
\text { variáveis }\end{array}$ \\
\hline
\end{tabular}

N/I- Não informado NS- Número de sessões DI - Duração da intervenção em minutos. GE- Grupo experimental GC- Grupo controle GP - Grupo placebo 
Quadro 2: estudos do therapeutic touch no câncer

\begin{tabular}{llll}
\hline \multicolumn{1}{c}{ Referência } & \multicolumn{1}{c}{ Técnica NS/DI/GE } & \multicolumn{1}{c}{ Uso } & \multicolumn{1}{c}{ Efeito } \\
\hline $\begin{array}{l}\text { Aghabati N; et } \\
\text { al 2010 }\end{array}$ & $\begin{array}{l}\text { TT NS-05/ DI-30 GE-30/ } \\
\text { GC-30/GP-30 }\end{array}$ & $\begin{array}{l}\text { Efeitos colaterais provenientes } \\
\text { da quimioterapia }\end{array}$ & $\begin{array}{l}\text { Dor e fadiga tiveram uma melhora } \\
\text { significativa no GE em relação ao } \\
\text { GP e GC }\end{array}$ \\
\hline $\begin{array}{l}\text { Frank LS; et al } \\
\mathbf{2 0 0 7}\end{array}$ & $\begin{array}{l}\text { TT NS-01/DI-10 GE-42/ } \\
\text { GP-40 }\end{array}$ & $\begin{array}{l}\text { Ansiedade e dor em mulheres } \\
\text { ao passarem por biópsia }\end{array}$ & Não houve diferença significativa \\
\hline $\begin{array}{l}\text { Kelly AE; et al } \\
\mathbf{2 0 0 4}\end{array}$ & TT NS-02/DI-10 GE-18 & $\begin{array}{l}\text { Mulheres com câncer de mama } \\
\text { e sintomas de stress e ansie- } \\
\text { dade }\end{array}$ & $\begin{array}{l}\text { Promoção de sentimentos de } \\
\text { calma, relaxamento, segurança, } \\
\text { conforto e melhora da atenção }\end{array}$ \\
\hline Hallet A 2004 & TT NS-N/I/DI-N/I GE-07 & $\begin{array}{l}\text { Falta de concentração mental } \\
\text { em pacientes com câncer }\end{array}$ & $\begin{array}{l}\text { Os relatos demonstram que o TT } \\
\text { promove claridade e foco. }\end{array}$ \\
\hline $\begin{array}{l}\text { Sá AC et al 2003 } \\
\text { TT NS-07/DI-N/I GE-N/I } \\
\text { et al } \\
\mathbf{1 9 9 9}\end{array}$ & $\begin{array}{l}\text { Efeitos colaterais da quimio- } \\
\text { terapia }\end{array}$ & $\begin{array}{l}\text { Melhora da dor, náusea e dos níveis } \\
\text { de hemoglobina }\end{array}$ \\
\hline
\end{tabular}

N/I- Não informado NS- Número de sessões DI - Duração da intervenção em minutos. GE- Grupo experimental GC- Grupo controle GP - Grupo placebo

Quadro 3: Estudos Do Healing Touch No Câncer

\begin{tabular}{llll}
\hline Referência & Técnica NS/DI/GE & Uso & Efeito \\
\hline FitzHenry 2013 & $\begin{array}{l}\text { HT NS- N/I DI-45 GE- 20 } \\
\text { GP-21 }\end{array}$ & $\begin{array}{l}\text { Fadiga em pacientes com câncer } \\
\text { de mama }\end{array}$ & Não houve diferença significativa \\
\hline $\begin{array}{l}\text { Wong J; et al } \\
\text { HT NS-03/DI-30 }\end{array}$ & $\begin{array}{l}\text { Crianças com câncer e sintomas } \\
\text { de dor, stress e fadiga. }\end{array}$ & $\begin{array}{l}\text { Melhora significativa de todas as } \\
\text { variáveis }\end{array}$ \\
$\begin{array}{l}\text { Judson PL; et al } \\
\mathbf{2 0 1 1}\end{array}$ & $\begin{array}{l}\text { HT NS-06/DI-30/GE-23/ } \\
\text { GC-20 }\end{array}$ & $\begin{array}{l}\text { Qualidade de vida em mulheres } \\
\text { com câncer de ovário }\end{array}$ & Não houve diferença significativa \\
\hline $\begin{array}{l}\text { Lutgendorf SK; } \\
\text { et al 2010 }\end{array}$ & $\begin{array}{l}\text { HT NS-24/DI-20 a 30 } \\
\text { GE-17/GC-17 }\end{array}$ & $\begin{array}{l}\text { Pessoas recebendo quimiote- } \\
\text { rapia }\end{array}$ & Não houve diferença significativa \\
\hline $\begin{array}{l}\text { Danhauer SC; } \\
\text { et al 2008 }\end{array}$ & HT NS-03/DI-30 GE-12 & $\begin{array}{l}\text { Pacientes com Leucemia e sinto- } \\
\text { mas de fadiga e náusea. }\end{array}$ & $\begin{array}{l}\text { Melhora significativa de todas as } \\
\text { variáveis }\end{array}$ \\
\hline $\begin{array}{l}\text { Post-White et al } \\
\mathbf{2 0 0 3}\end{array}$ & $\begin{array}{l}\text { HT NS-04/DI-45 } \\
\text { GE-56/GC-108 }\end{array}$ & $\begin{array}{l}\text { Pessoas com câncer de qualquer } \\
\text { tipo }\end{array}$ & $\begin{array}{l}\text { Diminuição da fadiga, P.A. } \\
\text { batimento cardíaco, frequência } \\
\text { respiratória e melhora do ânimo. }\end{array}$ \\
\hline
\end{tabular}

N/I- Não informado NS- Número de sessões DI - Duração da intervenção em minutos. GE- Grupo experimental GC- Grupo controle GP - Grupo placebo

\section{DISCUSSÃO}

A pesquisa das TIM no câncer se baseia única e exclusivamente na sua utilização como recurso complementar em adição ao tratamento convencional. Não existem dados científicos que confirmem que estas técnicas não possam ser utilizadas como recurso alternativo, assim partir do pressuposto de que elas curam ou não o câncer é basear-se em pré-noções.
Outro possível limite que a maioria dos estudos apresenta é relativo à padronização da técnica, pois dos 15 estudos que apresentam protocolo associado à aplicação das TIM, 7 deles obtiveram resultados não significativos enquanto 8 resultados significativos. Entretanto nos 4 estudos que utilizaram uma abordagem individual e não padronizada, todos tiveram resultados significativos, o que indica que os efeitos dessas técnicas são melhor percebidos pelos 
pacientes e efetuados pelos terapeutas sob abordagem fenomenológica.

Dos 12 estudos que relatam a formação do terapeuta que aplicou a técnica, 7 foram de terapeutas experientes, sendo 4 com resultados significativos e 3 não significativos, e 5 de terapeutas inexperientes, sendo que apenas 1 com resultado significativo e 4 não significativos. Porém, devido ao pequeno número de artigos que descrevem este dado, não é possível tirar conclusões se o nível de experiência dos praticantes interferiu nos resultados.

O tempo de intervenção e número de sessões, aparentemente, não influenciou o resultado dos estudos. $\mathrm{O}$ que destoa das conclusões de Lafreniere et $\mathrm{a}^{35}$ que as TIM têm efeito acumulativo, pois em seu artigo os melhores resultados foram obtidos na última sessão.

Apenas 3 estudos utilizaram grupo placebo, todos com resultados não significativos. Em estudos como estes, o uso das TIM tem sido criticado em relação aos resultados alcançados, pois segundo alguns pesquisadores provêm apenas do efeito placebo. No entanto, em grande parte das vezes, a metodologia destes estudos atende ao modelo de estudos clínicos randomizados, excluindo o valor da interação terapeuta-paciente e a intencionalidade do terapeuta constituindo, assim, um limite das pesquisas ${ }^{37}$.

A secundarização da metodologia qualitativa dificulta a criação de evidências do uso das TIM no tratamento do câncer, criando lacunas neste campo de conhecimento. É necessário expandir as fronteiras da ciência para que a pesquisa em TIM se alinhe ao modelo de cuidado convencional. É imprescindível compreender que na pesquisa ortodoxa ou não-ortodoxa, 0 método científico é valorizado pelo seu rigor, porém as evidências têm sido rejeitadas ou confirmadas de acordo com a visão de mundo da comunidade acadêmica ${ }^{18}$.

Em relação ao suposto efeito placebo que as TIM possam apresentar, é importante salientar que por serem terapias que não utilizam nenhum arcabouço inerente ao imaginário da medicina convencional, têm um efeito psicológico muito mais brando comparadas a outras PIC, como a acupuntura e a fitoterapia, que utilizam instrumentos consagrados no imaginário biomédico como fármacos e agulhas.
Este imaginário biomédico criou e é recriado pela Medicina Baseada em Evidência (MBE), a qual modela: a forma dos serviços de saúde, os fundos de saúde e as políticas, práticas e modelos de cuidado. Assim, estipulam qual tratamento é válido, os caracteres da doença, qualidade de vida, e ultimamente as chances de sobrevivência ${ }^{38}$. No entanto, isto ocorre também no universo das práticas não convencionais, pois apesar de o Reiki ser a PIC mais utilizada no SUS em 2004, ele não entrou na PNPIC em 2006, pelo motivo de esta técnica não ter evidências suficientes ${ }^{7}$.

A Prática Baseada em Evidências (PBE) surgiu a partir da necessidade de atualização e de confiabilidade das práticas clínicas, buscando estabelecer um elo entre pesquisa e prática clínica e os ensaios clínicos randomizados são considerados o padrão ouro para confiabilidade dos resultados. Se os estudos quantitativos respondem melhor a questões de pesquisa que buscam a causa, prognóstico, diagnóstico, prevenção, tratamento ou custos sobre saúde, a pesquisa qualitativa responde melhor a questões que sugerem reflexões mais subjetivas, sobre percepções, significações, experiências sobre doença, sentimentos e emoções dos pacientes sobre os efeitos de alguma técnica. Portanto, para diminuir a polarização cultural na construção do que seria um conhecimento legítimo sobre os efeitos de práticas energéticas como as TIM, novos instrumentos de avaliação e diferentes tipos de delineamento de pesquisa precisam ser estudados e propostos. $1^{18,38}$.

Um dos limites deste trabalho consiste no fato de a maioria dos estudos serem aplicados a pacientes com câncer com diversos sintomas e aspectos diferentes associados a esta doença, o que dificulta a avaliação objetiva das TIM no câncer. Outro limite é relativo ao número reduzido de artigos indexados identificados e analisados, devido ao fato de este campo de pesquisa ser bastante novo.

\section{CONCLUSÃO}

Este estudo promove um vislumbre dos possíveis efeitos benéficos das TIM no cuidado de pessoas em tratamento do câncer, assim como os limites causa- 
dos por modelos de pesquisa provenientes de estudos quase exclusivamente quantitativos.

Há evidências de que a pesquisa das TIM no câncer destoa da lógica biomédica, e a validação de abordagem fenomenológica é um ponto crucial a ser desenvolvido para que haja uma melhor compreensão deste tema. Assim, futuros estudos devem incluir mensurações qualitativas que deem voz aos terapeutas e pacientes, para compreender como eles experimentam essas técnicas, como elas podem colaborar para a melhora da qualidade de vida do paciente oncológico, bem- estar e outras variáveis indispensáveis para o enfrentamento de tratamentos longos e difíceis.

\section{CONFLITOS DE INTERESSE}

declararam não haver

\section{FONTES DE FINANCIAMENTO}

nenhuma

\section{REFERÊNCIAS}

1. VanderVaart S, Berger H, Tam C, Goh YI, Gijsen VM, de Wildt SN, Taddio A, Koren $\mathrm{G}$. The effect of distant reiki on pain in women after elective Caesarean section: a double-blinded randomized controlled trial. BMJ open 2011; 1(1):e000021

2. VanderVaart S, Gijsen VM, Wildt SN, Koren G. A systematic review of the therapeutic effects of reiki.J Altern Complement Med 2009; 15 (11): 1157-69.

3. Babenko PC. Reiki: um estudo localizado sobre terapias alternativas, ideologia e estilo de vida. [Dissertação] São Carlos (SP) Universidade Federal de São Carlos; 2004.

4. Stewart JC. The Reiki Touch: The essential Handbook. Houston The Reiki Touch: 1995.

5. Usui M, Petter FA. Manual de Reiki do Dr. Mikao Usui. 13 ${ }^{\mathrm{a}}$ ed. São Paulo: Pensamento; 1999

6. National Center of Complementary and Alternative Medicine. Estados Unidos da América: NCCAM. US National Institutes of Health. 2013. [Acesso em 2013 out 19]. Disponível em http:// nccam.nih.gov/health/reiki/introduction.htm?nav=gsa.

7. Brasil. Portaria ${ }^{\circ} 971$ de 03 de maio de 2006. Aprova a Política Nacional de Práticas Integrativas e Complementares (PNPIC) no Sistema Único de Saúde. Ministério da Saúde 2006; 03 mai.

8. Gerber R. Um guia prático de medicina vibracional. In: Gerber R. As variedades da cura pelas mãos. São Paulo: Cultrix: 2001.p. 393-425.

9. Krieger D. Therapeutic touch: the Imprimatur of Nursing. Am J Nurs 1975. 35 (5): 784-787.

10. Marta IER. Utilização do toque terapêutico no processo de cuidar de pessoas com dor crônica não-neoplásica [Tese] Ribeirão Preto (SP). Escola de Enfermagem de Ribeirão Preto; 1999.

11. Oliveira VLM. O toque terapêutico no alívio da dor e seu desconforto em doentes vasculares amputados com enfermidades arteriais de membros inferiores [Dissertação] Guarulhos (SP). Universidade Guarulhos; 2000.

12. Post-White J. Kinney ME, Savik KGAUJB, Wilcox C, Lerner I. Therapeutic Massage and Healing Touch Improve Symptons in Cancer. Integr Cancer Ther 2003; 2 (4):322-344.
As TIM colaboram com a ecologia dos saberes, ao ampliar o escopo de atuação terapêutica e incluir formas integrativas e complementares de tratamento que podem promover saúde, pois permitem a manutenção e melhora da qualidade de vida com técnicas não invasivas, de baixo custo e com pequenos registros de contraindicações em pacientes que passam pela severidade desta doença. Colaboram, também, com dados que contribuem com a PNPIC no aspecto de integralidade do cuidado ao paciente oncológico ao tratarem-no de maneira multidimensional, com uma abordagem centrada na pessoa, considerando dimensões importantes que vão além do biológico e da doença, como por exemplo, o campo de energia.

13. Peters RM. The effectiveness of Therapeutic Touch: A meta-analytic review. Nurs Sci Q 1999; 12 (1), 52-61.

14. Spadacio, C, Barros, NF. O uso de medicinas alternativas e complementares por pacientes com câncer: revisão sistemática. Rev Saúde Públ 2008 42(1): 158-64

15. Beard C, Stason WB, Wang Q, Manola J, Dean-Clower E, Dusek JA, Decristofaro S, Webster A, Doherty-Gilman AM, Rosenthal DS, Benson H. Effects of Complementary Therapies on Clinical Outcomes in Patients Being Treated with Radiation Therapy for Prostate Cancer. Cancer 2011; 117(1): 96-102.

16. Tovey P, Barros NF, Hoehne EL, Carvalheira JBC. Use of Traditional Medicine and Globalized Complementary and Alternative Medicine Among Low-Income Cancer Service Users in Brazil. Integr Cancer Ther 2006; 5(3): 232-5.

17. Gonçalo CS, Castro CM, Bonon MM, Motta PMR, Dahdal AB, Batista JC, Hirayama MS, Peres SMP, Barros NF. Planejamento e execução de Revisões Sistemáticas da Literatura. Brasília Médica 2012; 49 (2).

18. Motta PMR, Tavares MM. Resenha do livro: Evidence Healthcare in Context - Critical Social Science Perspectives. Cad Saúde Pública No prelo 2013

19. Thomso Reuters. Journal Citation Reports. EUA; 2013. [acesso em 20 de Outubro de 2013] Diponível em: http://thomsonreuters.com/ journal-citation-reports/

20. Kelly AE, Sullivan P, Fawcet J, Samarel N. Therapeutic Touch, Quiet Time, and Dialogue: Perceptions of Women With Breast Cancer. Oncol Nurs Forum 2004; 31(2): 625-631.

21. Catlin A, Taylor-Ford R, Investigation of Standard Care Versus Sham Reiki Placebo Versus Actual Reiki Therapy to Enhance Comfort and Well-Being in a Chemotherapy Infusion Center. Oncol Nurs Forum 2011; 38(3): 212-20.

22. Olson K, Hanson J, Michaud M. A Phase II Trial of Reiki for the Management of Pain in Advanced Cancer Patients.J Pain Simptom Manage 2003; 26(5): 990-97.

23. Fleisher KA, Mackenzie ER, Frankel ES, Seluzicki C, Casarett D, Mao JJ. Integrative Reiki for Cancer Patients: A Program Evaluation. Integr Cancer Ther 2013; 20(10): 1-6. 
24. FitzHenry F, Wells N, Slater V, Dietrich MS, Wisawatapnimit P, Chakravarthy AB. A Randomized Placebo-Controlled Pilot Study of the Impact of Healing Touch on Fatigue in Breast Cancer Patients Undergoing Radiation Therapy. Integr Cancer Ther 2013; 20(10): $1-9$.

25. Wong J, Ghiasuddin A, Kimata C, Patelesio B, Siu A. The Impact of Healing Touch on Pediatric Oncology Patients. Integr Cancer Ther 2012; 20(10): 1-4

26. Tsang KL, Carlson LE, Olson K. Pilot crossover Trial of Reiki versus rest for treating câncer-related fatigue. Integr Cancer Ther 2007; 25(6): 25-35.

27. Frank, LS; Frank JL; March D; Makari-Judson G; Barham RB; Mertens WC. Does therapeutic Touch Ease the discomfort or distress of patients undergoing stereotactic core breast biopsy? A randomized clinical trial. Pain Medicine 2007; 8(5): 419-424.

28. Marcus DA, O’Neill BB, Kopar JL. Symptomatic Improvement Reported After Receiving Reiki at a Cancer Infusion Center. Am J Hosp Palliat Care 2013; 30(2): 216-217.

29. Judson PL, Dickson EL, Argenta PA, Xiong Y, Geller MA, Carson LF, Ghebre R, Jonson AL, Junior LSD. A prospective randomized tria of integrative medicine for women with ovarian cancer. Ginecol Oncol 2011; 123(2): 346-350.

30. Lutgendorf SK, Mullen-Houser E, Russel D, DeGeest K, Jacobson G, Hart L, Bender D, Anderson B, Buekers TE, Goodheart MJ, Antoni MH, Sood AK, Lubaroff DM. Preservation of immune function in cervical cancer patients during chemoradiation using a novel integrative approach. Brain Behav Immun 2010; 24(8): 1231-1240.
31. Potter PJ. Breast Biopsy and distress: Feasibility of Testing a Reiki Intervention. J Holist Nurs 2007; 25(4): 238-248.

32. Aghabati N, Mohammadi E, Esmaiel P. The effect of Therapeutic Touch on Pain and Fatigue of Cancer Patients Undergoing Chemotherapy. eCAM 2010; 7(3): 375-381.

33. Sá AC, Silva MJP. Aplicação do toque terapêutico em mulheres portadoras de câncer de mama sob tratamento quimioterápico. Mundo Saúde 2010; 27(2): 258-269.

34. Danhauer SC, Tooze JÁ, Holder P, Miller C, Jesse MT, Carrol S, Larrimore D, Campbell CR, Kemper KJ. Healing Touch as a Supportive Intervention for Adult Acute Leukemia Patients: A Pilot Inverstigation of Effects on Distress and Symptoms. J Soc Integr Oncol 2008; 6(3): 89-97.

35. Lafreniere KD; Mutus B; Cameron S; Tannous M; Giannotti M Abu-Zahra H; Laukkanen E. Effects of Therapeutic Touch on Biochemical and Mood Indicators in Women. The J. of Alter. And Compl. Med 1999; 5(4): 367-370.

36. Hallet A. Narratives of Therapeutic Touch. Nurs Stand 2004; 19(1): 33-37

37. Larden CN, Palmer ML, Janssen P. Efficacy of Therapeutic Touch in Treating Pregnant Impatients Who Have a Chemical Dependecy. $J$ Holist Nurs 2004; 22(4): 320-332.

38. Broom A, Adams J. Evidence-Based Healthcare in Context Critical Social Science Perspective. $1^{\mathrm{a}}$ ed. Inglaterra: Ashgate; 2012. 